Research Paper:

\title{
Recombinant Rabies Virus With a Multiple Cloning Site: A Platform Capable of Immunological Alterations
}

\author{
Seyed Hamidreza Naghi Mousavi ${ }^{1}$ (D), Alireza Gholami2 ${ }^{*}$ (D), Mojtaba Jafarinia ${ }^{1}$, Mehdi Ajorloo ${ }^{3,4^{*}}$ (D) \\ 1. Department of Biology, Marvdasht Branch, Islamic Azad University, Marvdasht, Iran. \\ 2. Viral Vaccines Production Unit, Research and Production Complex, Pasteur Institute of Iran, Tehran, Iran. \\ 3. Hepatitis Research Center, Lorestan University of Medical Sciences, Khorramabad, Iran. \\ 4. Department of Clinical Laboratory Sciences, School of Allied Medical Sciences, Lorestan University of Medical Sciences, Khorramabad, Iran
}

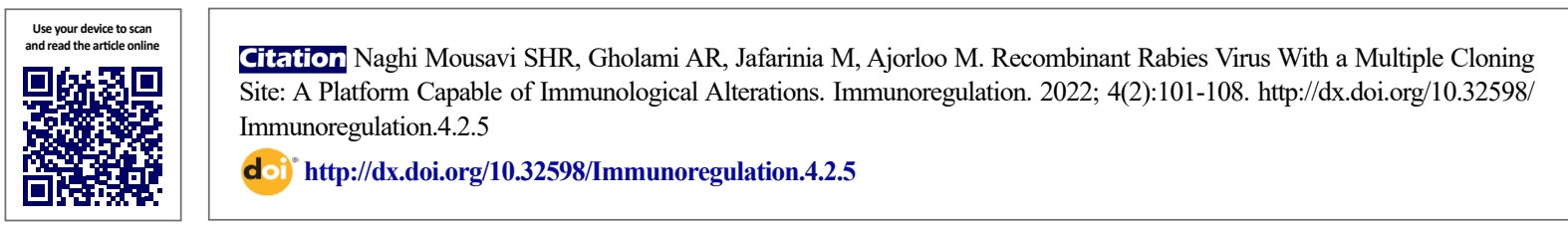

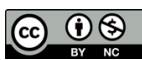

Article info:

Received: 22 Mar 2021

Accepted: 21 Jun 2021

Available Online: 01 Jan 2022

Keywords:

Rabies virus, Reverse genetics, Virus engineering, Molecular cloning

\begin{abstract}
A B S T RA C T
Background: Rabies is fatal encephalitis, i.e., preventable by appropriate vaccination. Reverse genetics has proved promising for manipulating the rabies virus immunological characteristics. The insertion or deletion of a gene from the rabies genome could render specific functions to the rabies virus.

Materials and Methods: A multiple cloning site including 111 nucleotides long harboring 10 singlecut restriction sites have been designed. The designed fragment was cloned between the $\mathrm{G}$ and $\mathrm{L}$ genes of the rabies virus genome. The recombinant rabies virus was rescued, and its infectivity was confirmed in the BHK-21 cell line. The recombinant virus propagation was compared with the initial rabies virus strain. Statistical analysis was performed using GraphPad Prism.

Results: The cloning and localization of the multiple cloning site were verified by nucleotide sequencing. The recombinant virus properly propagated and rescued in the BHK-21 cell line. Comparing the recombinant virus with the initial rabies virus has shown that both viruses had similar functionality and propagation rate.

Conclusion: The recombinant virus obtained in the present study could facilitate further cloning experiments. Examples include constructing a marker virus, carrying green fluorescent protein to be used either in rabies immunity assays or tracking the virus infection in relevant tissues.
\end{abstract}

* Corresponding Author:

Alireza Gholami, PhD.

Address: Viral Vaccines Production Unit, Research and Production Complex, Pasteur Institute of Iran, Tehran, Iran

Phone: +98 (912) 3486080

E-mail: a.gholami@pasteurac.ir
Mehdi Ajorloo, PhD.

Address: Hepatitis Research Center, Lorestan University of Medical Sciences, Khorramabad, Iran.

Phone: +98 (912) 5686632

E-mail:kmehdiajorloo@gmail.com 


\section{Introduction}

abies is the deadliest zoonotic viral disease caused by the rabies virus. The $\mathbf{R}$ virus is transmitted through bites or scratches in the saliva of a rabid animal to other animals and individuals. It is neglected and underreported; however, according to data, rabies is found in 5 continents, causing over 59000 human deaths annually. Children under 15 years old are victims of $40 \%$ of global deaths from rabies. Moreover, $>95 \%$ of victims belong to Asia and Africa [1]. Rabies is $100 \%$ fatal after clinical symptoms appear. However, it is a vaccine-preventable infection. Still, effective vaccines and immunoglobulins are not easily accessible in underprivileged areas where more needed. Besides, the incidence of rabies is high [2]. Post-exposure prophylaxis after rabies exposure seems to cost on average US\$ 108 per person, i.e., unaffordable in the areas mentioned above [3]. The restraining of rabies spread is feasible through One-Health approaches engaging human-animal-environment sectors in the control campaigns. Additionally, surveillance and monitoring are necessary elements that should be in place based on laboratory assays and diagnosis tests $[4,5]$. A critical assay to monitor the level of immunity in individuals and animals is the Rapid Fluorescent Focus Inhibition Test (RFFIT). This test is based on the seroneutralization concept in mammalian cell culture, using rabies virus and neutralizing serum. A fluorescent antibody would later be applied to detect the virus-infected cells and quantify the results [6]. Employing a fluorescent virus would make the use of the antibody dispensable. Previous studies indicated successful insertion and utilization of a Green Fluorescent Protein (GFP) carrying rabies virus in laboratory assays $[7,8]$.

Rabies virus belongs to the genus Lyssavirus and the family Rhabdoviridae. The rabies virus has a helical Ribonucleoprotein (RNP) core, consisting of a non-segmented single-stranded RNA genome of $12 \mathrm{~Kb}$ length, coated with the virus Nucleoprotein $(\mathrm{N})$. Other virus proteins include Phosphoprotein (P), Matrix protein (M), Glycoprotein $(\mathrm{G})$, and the Large (L) or polymerase protein. There is also a non-coding pseudogene region between G and L genes [9]. All the proteins are necessary for the viral life cycle; however, the polymerase complex, which is comprised of N, P, and L, is sufficient for genome replication and expression. This is the concept of rabies virus formation by reverse genetics technology $[10,11]$ The reverse genetic technology provides the means to manipulate the genetic material of both DNA and RNA viruses and renders new phenotypes to those viruses. The genetic manipulation of organisms with reverse genetics and studying their phenotypic variations has become one of the fundamental techniques of modern molecular biology. However, the practice of reverse genetic techniques in negative-stranded RNA viruses has been more challenging due to their distinct replication cycle [12]. This study presented an approach to obtain a recombinant rabies virus containing a multiple cloning site which could facilitate further genetic manipulation of the virus.

\section{Materials and Methods}

The genome constructs required for expression of the polymerase complex and rescue the rabies virus consisted of plasmids carrying the rabies virus entire genome and plasmids encoding either P, N, or L genes. Those plasmids were obtained from the Pasteur Virus (PV) strain of the rabies virus. All plasmids include T7 promoters expressed in T7 RNA polymerase expressing Baby Hamster Kidney cell line (T7BHK). Those plasmids and T7BHK cell lines were provided by the viral vaccines production unit of Pasteur institute of Iran [13] A non-recombinant entire genome of the virus was used to control during all the procedures. A Multiple Cloning Site (MCS) containing ten unique restriction sites not existing in the rabies virus genome was designed using SnapGene software (Figure 1). The construct was obtained by nucleotide synthesis (GeneScript, USA), and the DraIII restriction site already located between $G$ and $\mathrm{L}$ genes was selected for cloning the fragment.

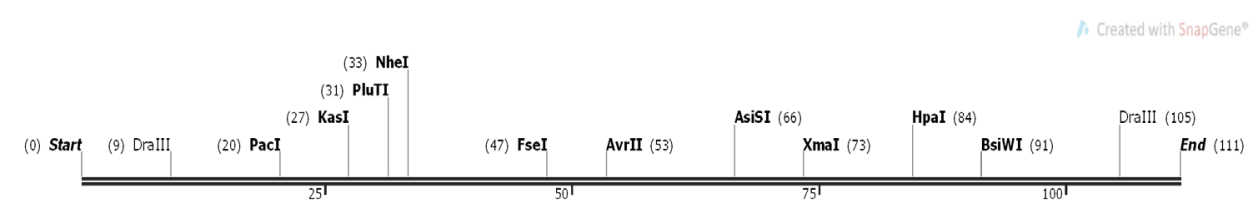

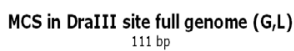

Figure 1. Multiple Cloning Site (MCS) contains ten restriction sites to insert the entire rabies genome 
The entire genome construct was partially digested using the DraIII enzyme (Thermo, USA) to obtain mono-digested forms of the complete genome. The linear structure of the full genome was resolved from other forms of the plasmid by running on the product of the digestion reaction on 0.5 percent agarose gel. The band for mono-digested forms of the complete genome was extracted from the gel using a gel extraction kit (GeneAl, Korea). Accordingly, the designed fragment carrying multiple cloning sites was digested by DraIII to prepare the fragment for the ligation reaction using T4 DNA ligase (Thermo, USA). Ecoli Top10 competent cell was used for transformation reaction conforming to standard ligation protocols [14]. Plasmid extraction from recombinant bacteria was performed using a miniprep plasmid extraction kit (YTA, Iran). Inserting the MCS in the virus genome was primarily verified through digesting the recombinant plasmid using the NheI enzyme (Thermo, USA). The sequence analysis of the recombinant plasmid was checked using designed primers (F: CTGACTGCCTTGATGTTGAT, R: CTCAGCCCTCTATTTCCTA).

The quantities of full recombinant genome required for the transfection step were obtained using the YTA Maxiprep Plasmid Extraction kit (YTA, Iran). The recombinant plasmid was transfected to the T7BHK cells using Lipofectamine ${ }^{\mathrm{TM}} 2000$ (Thermo, USA), conforming to the manufacturer's instructions. The T7BHK cells were co-transfected with $\mathrm{N}, \mathrm{P}$, and L coding plasmid constructs and the entire recombinant genome to form the polymerase complex and subsequent rescuing of the virus. The rescued rabies virus was released in the supernatant of the T7BHK cells, i.e., used to infect the BHK21 cell line. The next day, cold acetone fixed infected cells and stained them using a FITC-conjugated anti-rabies neucleocapsid antibody (Bio-Rad, USA). Cells were studied using an inverted fluorescent microscope (Motic, PRC) for the presence of rabies antigens. The RNA of the obtained virus was extracted using an RNA extraction kit (Cinnagen, Iran), and the sequence of the cloning site was re-checked using the primers mentioned above. During the rescue of the rabies virus, a non-recombinant rabies virus was also rescued in parallel. Titration of both the rescued viruses was performed using Nunc ${ }^{\mathrm{TM}} \mathrm{Lab}-$ Tek $^{\mathrm{TM}}$ chamber slides (Thermo, USA) and a FITC-conjugated anti-rabies neucleocapsid antibody (Bio-Rad, USA)in 8 repeats, according to standard protocols [6]. The titers were compared by statistical analyses using GraphPad Prism and the Mann-Whitney U test.

\section{Results}

Inserting the designed MCS in the rabies virus full genome construct between $\mathrm{G}$ and $\mathrm{L}$ genes was verified by PCR utilizing the primer pair mentioned in the previous section. The PCR reaction resulted in a $110 \mathrm{nt}$ larger product for successful cloning. The recombinant plasmid was also checked for the existence of the inserted MCS through enzymatic digestion using the NheI restriction enzyme. Sequence analysis demonstrated the correct nucleotide sequences and position of the MCS in the rescued virus genome. The results acquired from sequencing were analyzed by employing Mega 5 software [15].

A Fluorescent Antibody Test (FAT) proved the presence of the recombinant virus per rescuing step was proved by FAT. The virus was successfully detected in T7BHK and BHK21 cells (Figure 2).

The titers of both rescued viruses, with and without MCS insert, are presented in Supplementary Table 1. Statistical analysis of virus titers by Mann-Whitney test, obtained from eight independent experiments, did not result in significant differences (mean $\mathrm{PV}=1.025 \times 10^{7}$,
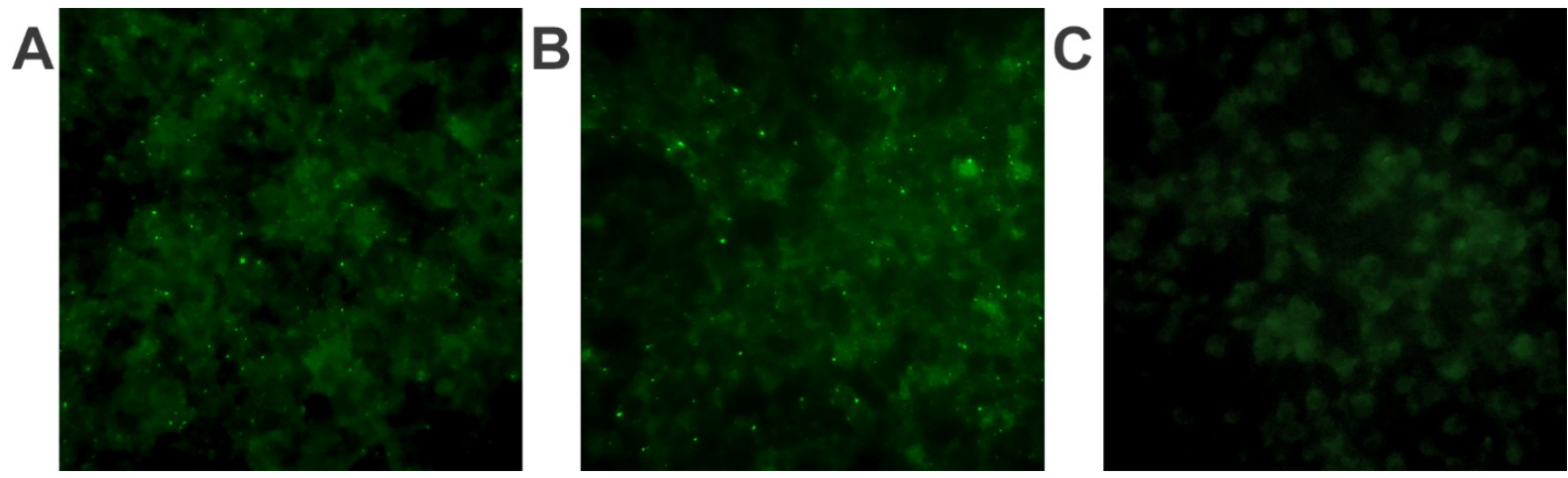

IMMUNoReGULATION

Figure 2. A Fluorescent Antibody Test (FAT) was used to verify the presence of rescued viruses in the BHK21 cell line

A: Non-recombinant Pasteur Virus (PV) strain; B: Recombinant virus comprised of PV strainwith MCS insert; C: Un-infected control cell. Apple green spots inside the cells are hallmarks of immunofluorescent staining of rabies virus protein-protein synthesis. 
Supplementary Table 1. The titers of PV and PV+MCS strains

\begin{tabular}{|c|c|c|}
\hline \multirow{2}{*}{ Experiment } & \multicolumn{2}{|c|}{ Virus Titers } \\
\hline & PV strain & $P V+$ MCS \\
\hline 1 & $8^{*} 10^{\wedge} 6$ & $2 * 10^{\wedge} 7$ \\
\hline 2 & $2 * 10^{\wedge} 7$ & $7^{*} 10^{\wedge} 6$ \\
\hline 3 & $3^{*} 10^{\wedge} 6$ & $1 * 10^{\wedge} 7$ \\
\hline 4 & $7^{*} 10^{\wedge} 6$ & $9^{*} 10^{\wedge} 6$ \\
\hline 5 & $2^{*} 10^{\wedge} 7$ & $4^{*} 10^{\wedge} 6$ \\
\hline 6 & $7^{*} 10^{\wedge} 6$ & $8^{*} 10^{\wedge} 6$ \\
\hline 7 & $7^{*} 10^{\wedge} 6$ & $6^{*} 10^{\wedge} 6$ \\
\hline 8 & $1 * 10^{\wedge} 7$ & $7^{*} 10^{\wedge} 6$ \\
\hline
\end{tabular}

mean $\left.\mathrm{PV}+\mathrm{MCS}=8.875 \times 10^{6}, \mathrm{P}=0.7694\right)$ (Supplementary Table $1 \&$ Figure 1$)$.

Those restriction sites were unique and already absent in the rabies virus genome. The sticky ends of DraIII sites at both ends of the fragment were specially designed to clone the fragment in the desired direction.

\section{Discussion}

The current study successfully designed, constructed, and inserted a multiple cloning site between the $G$ and $\mathrm{L}$ intergenic region of the Rabies Virus (RV) genome, facilitating further genetic manipulation of the virus. The presence of this multiple cloning site has been proved in the genome of the rescued rabies virus; however, it has not significantly affected the viral growth and amplifi- cation (Supplementary Table 1). Rabies encephalitis is caused by the rabies virus, a neurotropic virus of genus lyssavirus and family Rhabdoviridae [16]. Individuals at high risk of exposure to the virus, including travelers to high-risk regions, individuals in contact with suspected animals, researchers working on rabies virus, and health staff, need to be appropriately immunized. Considering the extremely high fatality of rabies encephalitis, the immunity against rabies should be verified in immunized people [2]. There exist numerous tests approved by the World Health Organization (WHO) to control rabies neutralizing antibody levels. The majority of those tests are based on a competitive attachment of the virus to a cell and Virus-Neutralizing Antibodies (VNAs).

Subsequently, an appropriate detection method would be necessary to demonstrate and interpret the test results.

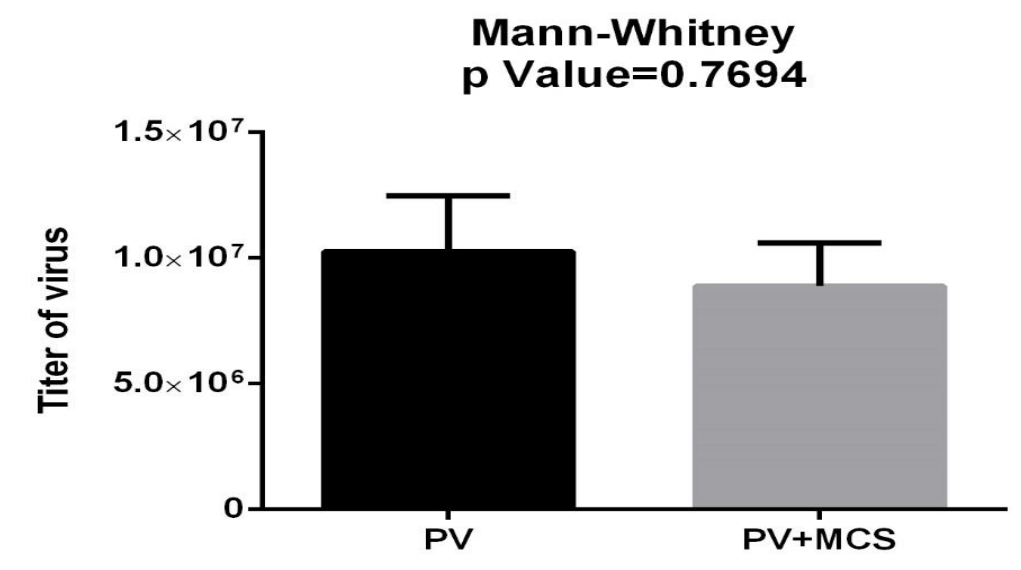

Supplementary Figure 1. The Mann-Whitney test revealed no significant differences between the strains 
One of the means of detection in those tests is utilizing an immunofluorescent antibody against the rabies virus used in the test. A previous study introduced a recombinant CTN181 strain of rabies virus expressing the green fluorescent protein GFP [17]. Using that recombinant virus, the researchers could monitor the growth of the virus in cell culture, independent of fluorescent staining. Reverse genetics technology has provided an efficient tool for manipulating the virus genome and constructing $\mathrm{RV}$ vectors carrying foreign genes [17].

The rescuing of the infectious RV from cloned cDNA was introduced in 1994 by Conzelmann and Schnell [18], which opened the possibility to various rabies virus proteins and cis-acting signals [11]. The reverse genetic studies on rabies virus included further research such as the anti-rabies virus drug profiles and inhibitors candidates [19]. It was also used to assess the immune response against a vaccine comprised of a recombinant rabies virus expressing the glycoprotein of the highly lethal Marburg virus [20].

The designed virus in this study has a Multiple Cloning Site (MCS) in the G-L intergenic region that renders the virus more flexible during genetic manipulations. This region has been recommended as the best location for cloning additional genes in the virus's genome [21-23]. Navid et al. compared two recombinant strains of rabies containing an additive copy of glycoprotein in two distinct locations of the genome with gene orders NPGMGL and NPMGGL. They found that the NPMGGL virus in which the additional $\mathrm{G}$ was located between $\mathrm{G}$ and $\mathrm{L}$ had higher virus titer and immunogenicity than the other [23].

Nevertheless, our results demonstrated that the recombinant rabies virus acquired from this study had no significantly different titers than the initial PV strain. In our study, the entire genome of the rabies virus carries two different sites for the DraIII restriction enzyme. The site between $G$ and L genes was chosen to insert the designed MCS [23].

A recombinant rabies virus carrying a GFP gene in a similar region has been used in the Rapid Fluorescent Focus Inhibition Test (RFFIT) and antibody titration methods [24]. In another study in 2011, a novel marker Gaussia luciferase (Gluc) was successfully cloned both into G-L and M-L Intergenic Regions (IGRs). In the present study, our results also comply with previous studies in which rescued viruses could correctly assemble and propagate, demonstrating that the MCS insertion in the G-L intergenic region had no adverse effect on virus packaging or propagation [17].
Generally, the CVS-11 strain of the rabies virus is applied in the RFFIT to quantify rabies neutralizing antibodies by the seroneuralization method [6]. The procurement of the fluorescent antibody for these methods could be resolved with such fluorescent viruses. Using GFP carrying recombinant CVS-11 in RFFIT has made the fluorescent antibody unrequired for the detection step of this test [24]. Accordingly, other strains of the rabies virus have been used to construct a GFP carrying virus used in the RFFIT test.

There are several reports about using Street Alabama Dufferin (SAD) B19 strain of the rabies virus as a successful viral vector for live vaccines against Human Immunodeficiency Virus/Simian Immunodeficiency Virus (HIV/SIV), Hepatitis C Virus (HCV), and Severe Acute Respiratory Syndrome CoV (SARS-CoV), or as delivery assistant for cytokines, cytochrome C, Tumor Necrosis Factor (TNF), and Interferon (IFN) [25-30]. In one study, a GFP was inserted between the nucleoprotein and the phosphoprotein of the RV's high egg passage Flury strain (HEP-Flury) [7]. There was no significant difference in growth kinetics between wild-type and recombinant viruses; however, the results suggested the high suitability of the N-P intergenic site for exogenous genes insertion and expression. An enhanced Green Fluorescent Protein (eGFP) fused to the SAD strain of RV phosphoprotein has been shown to render the labeling quality to the virus, enabling fluorescence labeling of live cells and tracking the virus [31]. Although a certain number of previous studies support protein insertion in the G-L region, similar qualities for tracking the $\mathrm{RV}$ in the recombinant strain, utilizing the MCS located in the G-L region, need to be studied with an independent set of complementary experiments.

\section{Conclusion}

In this study, the PV strain of the rabies virus was used to construct an MCS carrying strain. This construct shall provide an appropriate tool for directly inserting the genes of interest into the virus's genome. It must be further verified whether or not this strain with a GFP gene insertion in the G-L region would be favorable for the aforementioned in vitro specifications.

\section{Ethical Considerations}

\section{Compliance with ethical guidelines}

There were no ethical considerations to be considered in this research. 


\section{Funding}

The study was funded by Pasteur Institute of Iran.

\section{Authors' contributions}

Conceptualization, methodology, investigation, and gholamioriginal draft: All authors; Writing - review \& editing and resources: Alireza Gholami and Mehdi Ajorloo; Conceptualization, methodology, supervision, writing-review and editing; Supervision: Mojtaba Jafarinia.

\section{Conflicts of interest}

The authors declared no conflict of interest.

\section{Acknowledgments}

The authors would like to thank Ashkan Alamdary for his valuable contribution to this work.

\section{References}

[1] Hampson K, Coudeville L, Lembo T, Sambo M, Kieffer A, Attlan $\mathrm{M}$, et al. Estimating the global burden of endemic canine rabies. PLoS Neglected Tropical Diseases. 2015; 9(4):e0003709. [DOI:10.1371/journal.pntd.0003709] [PMID] [PMCID]

[2] Ertl HCJ. New rabies vaccines for use in humans. Vaccines. 2019; 7(2):54. [DOI:10.3390/vaccines7020054] [PMID] [PMCID]

[3] WHO/Department of control of neglected tropical diseases. Zero by 30: The global strategic plan to end human deaths from dog-mediated rabies by 2030 [Internet]. 2018 [Updated 2018 August 28]. Available from: https://apps.who.int/rabies/resources/9789241513838/en/index.html

[4] Acharya KP, Acharya N, Phuyal S, Upadhyaya M, Lasee S. One-health approach: A best possible way to control rabies. One Health. 2020; 10:100161. [DOI:10.1016/j.onehlt.2020.100161] [PMID] [PMCID]

[5] Acharya KP, Subedi D, Wilson RT. Rabies control in South Asia requires a One Health approach. One Health. 2021; 12:100215. [DOI:10.1016/j.onehlt.2021.100215] [PMID] [PMCID]

[6] World Health Organization, Rupprecht, Charles E, Fooks, Anthony R Abela-Ridder, Bernadette. Laboratory techniques in rabies. volume $1,5^{\text {th }}$ ed. World Health Organization. https://apps.who.int/iris/handle/10665/310836.

[7] Luo J, Zhao J, Tian Q, Mo W, Wang $Y$, Chen $H$, et al. A recombinant rabies virus carrying GFP between $\mathrm{N}$ and $\mathrm{P}$ affects viral transcription in vitro. Virus Genes. 2016; 52(3):379-87. [DOI:10.1007/s11262-016-1313-2] [PMID] [PMCID]

[8] Yang DK, Kim HH, Park YR, Yoo JY, Park Y, Park J, et al. Generation of a recombinant rabies virus expressing green fluorescent protein for a virus neutralization antibody assay. Journal of Veterinary Science. 2021; 22(4):e56. [DOI:10.4142/ jvs.2021.22.e56] [PMID] [PMCID]
[9] Banyard AC, Tordo N. Rabies pathogenesis and immunology. Revue Scientifique et Technique. 2018; 37(2):323-30. [DOI:10.20506/rst.37.2.2805] [PMID]

[10] Mebatsion T, Schnell MJ, Cox JH, Finke S, Conzelmann KK. Highly stable expression of a foreign gene from rabies virus vectors. Proceedings of the National Academy of Sciences of the United States of America. 1996; 93(14):7310-4. [DOI:10.1073/pnas.93.14.7310] [PMID] [PMCID]

[11] Scher G, Schnell MJ. Rhabdoviruses as vectors for vaccines and therapeutics. Current Opinion in Virology. 2020; 44:169-82. [DOI:10.1016/j.coviro.2020.09.003] [PMID] [PMCID]

[12] Osakada F, Callaway EM. Design and generation of recombinant rabies virus vectors. Nature Protocols. 2013; 8(8):1583-601. [DOI:10.1038/nprot.2013.094] [PMID] [PMCID]

[13] Ajorloo M, Bamdad T, Gholami AR, Azadmanesh K. Assessment the efficiency of the constructed minigenome of rabies virus using PV strain as helper virus. Archives of Iranian Medicine. 2016; 19(5):335-41. [PMID]

[14] Zhao F, Ying Zhang Y, Li H, Shi RJ, Han SQ. [CaCl2-heat shock preparation of competent cells of three Pseudomonas strains and related transformation conditions (Chinese)] Ying Yong Sheng Tai Xue Bao. 2013; 24(3):788-94. [PMID]

[15] Tamura K, Peterson D, Peterson N, Stecher G, Nei M, Kumar S. MEGA5: Molecular evolutionary genetics analysis using maximum likelihood, evolutionary distance, and maximum parsimony methods. Molecular Biology and Evolution. 2011; 28(10):2731-9. [DOI:10.1093/molbev/msr121] [PMID] [PMCID]

[16] Yin C. Progress in the development of animal rabies vaccines in China. China CDC Weekly. 2021; 3(39):825-30. [DOI:10.46234/ccdcw2021.204] [PMID] [PMCID]

[17] Du J, Tang Q, Huang Y, Rodney WE, Wang L, Liang G Development of recombinant rabies viruses vectors with Gaussia luciferase reporter based on Chinese vaccine strain CTN181. Virus Research. 2011; 160(1-2):82-8. [DOI:10.1016/j. virusres.2011.05.018] [PMID] [PMCID]

[18] Conzelmann KK, Schnell M. Rescue of synthetic genomic RNA analogs of rabies virus by plasmid-encoded proteins. Journal of Virology. 1994; 68(2):713-9. [DOI:10.1128/ jvi.68.2.713-719.1994] [PMID] [PMCID]

[19] Du Pont V, Plemper RK, Schnell MJ. Status of antiviral therapeutics against rabies virus and related emerging lyssaviruses. Current Opinion in Virology. 2019; 35:1-13. [DOI:10.1016/j.coviro.2018.12.009] [PMID] [PMCID]

[20] Keshwara R, Hagen KR, Abreu-Mota T, Papaneri AB, Liu D, Wirblich C, et al. A Recombinant Rabies virus expressing the marburg virus glycoprotein is dependent upon antibody-mediated cellular cytotoxicity for protection against marburg virus disease in a murine model. Journal of $\mathrm{Vi}$ rology. 2019; 93(6):e01865-18. [DOI:10.1128/JVI.01865-18] [PMID] [PMCID]

[21] Liu X, Yang Y, Sun Z, Chen J, Ai J, Dun C, et al. A recombinant rabies virus encoding two copies of the glycoprotein gene confers protection in dogs against a virulent challenge. PLoS One. 2014; 9(2):e87105. [DOI:10.1371/journal. pone.0087105] [PMID] [PMCID]

[22] Faber M, Pulmanausahakul R, Hodawadekar SS, Spitsin S, McGettigan JP, Schnell MJ, et al. Overexpression of the 
rabies virus glycoprotein results in enhancement of apoptosis and antiviral immune response. Journal of Virology. 2002; 76(7):3374-81. [DOI:10.1128/JVI.76.7.3374-3381.2002] [PMID] [PMCID]

[23] Navid MT, Li Y, Zhou M, Cui M, Fu ZF, Tang L, et al. Comparison of the immunogenicity of two inactivated recombinant rabies viruses overexpressing the glycoprotein. Archives of Virology. 2016; 161(10):2863-70. [DOI:10.1007/ s00705-016-2967-8] [PMID]

[24] Huang Y, Tang Q, Nadin-Davis SA, Zhang S, Hooper CD, Ming $P$, et al. Development of a reverse genetics system for a human rabies virus vaccine strain employed in China. Virus Research. 2010; 149(1):28-35. [DOI:10.1016/j.virusres.2009.12.009] [PMID]

[25] Faber M, Lamirande EW, Roberts A, Rice AB, Koprowski $\mathrm{H}$, Dietzschold B, et al. A single immunization with a rhabdovirus-based vector expressing severe acute respiratory syndrome coronavirus (SARS-CoV) $S$ protein results in the production of high levels of SARS-CoV-neutralizing antibodies. The Journal of General Virology. 2005; 86(Pt 5):1435-40. [DOI:10.1099/vir.0.80844-0] [PMID] [PMCID]

[26] Faber M, Bette M, Preuss MAR, Pulmanausahakul R, Rehnelt J, Schnell MJ, et al. Overexpression of tumor necrosis factor alpha by a recombinant rabies virus attenuates replication in neurons and prevents lethal infection in mice. Journal of Virolology. 2005; 79(24):15405-16. [DOI:10.1128/ JVI.79.24.15405-15416.2005] [PMID] [PMCID]

[27] Pulmanausahakul R, Faber M, Morimoto K, Spitsin S, Weihe E, Hooper DC, et al. Overexpression of cytochrome C by a recombinant rabies virus attenuates pathogenicity and enhances antiviral immunity. Journal of Virolology. 2001; 75(22):10800-7. [DOI:10.1128/JVI.75.22.10800-10807.2001] [PMID] [PMCID]

[28] Faul EJ, Aye PP, Papaneri AB, Pahar B, McGettigan JP, Schiro F, et al. Rabies virus-based vaccines elicit neutralizing antibodies, poly-functional CD8+ T cell, and protect rhesus macaques from AIDS-like disease after SIV(mac251) challenge. Vaccine. 2009; 28(2):299-308. [DOI:10.1016/j.vaccine.2009.10.051] [PMID] [PMCID]

[29] Zhao L, Toriumi H, Kuang Y, Chen H, Fu ZF. The roles of chemokines in rabies virus infection: Overexpression may not always be beneficial. Journal of Virology. 2009; 83(22):11808-18. [DOI:10.1128/JVI.01346-09] [PMID] [PMCID]

[30] Kuang Y, Lackay SN, Zhao L, Fu ZF. Role of chemokines in the enhancement of BBB permeability and inflammatory infiltration after rabies virus infection. Virus Research. 2009; 144(1-2):18-26. [DOI:10.1016/j.virusres.2009.03.014] [PMID] [PMCID]

[31] Finke S, Brzozka K, Conzelmann KK. Tracking fluorescence-labeled rabies virus: Enhanced green fluorescent protein-tagged phosphoprotein $\mathrm{P}$ supports virus gene expression and formation of infectious particles. Journal of $\mathrm{Vi}-$ rology. 2004; 78(22):12333-43. [DOI:10.1128/JVI.78.22.1233312343.2004] [PMID] [PMCID] 
This Page Intentionally Left Blank 